\title{
Publisher Correction: Stand-off trapping and manipulation of sub-10 nm objects and biomolecules using opto-thermo-electrohydrodynamic tweezers
}

Chuchuan Hong, Sen Yang and Justus C. Ndukaife iD

Correction to: Nature Nanotechnology https://doi.org/10.1038/s41565-020-0760-z, published online 31 August 2020.

An incorrect version of Supplementary Video 4 was originally published for this Letter; both PS structures were labelled '20 nm PS', but the second should have been ' 100 nm PS'. The video has now been replaced with the correct version.

Published online: 29 September 2020

https://doi.org/10.1038/s41565-020-00786-Z

๑ The Author(s), under exclusive licence to Springer Nature Limited 2020 